\title{
PRELIMINARY TESTING AND ANALYSIS OF AN OPTIMIZED ROBOTIC ARM, FOR CT IMAGE GUIDED MEDICAL PROCEDURES.
}

\section{GOURISHANKAR MOHAPATRO, RUBY MISHRA \& SHAH SHUBHAM KAMLESH}

School of Mechanical Engineering, Kiit University, Bhubaneswar, Odisha, India

In recent times of evidence based accurate diagnosis. Tissue sampling is done under CT guidance to obtain the accurate diagnosis under specific circumstances. But currently there is no accurate method to do such diagnosis as doctors still use eye ball method to direct the needle using CT image. In this research work an optimized robotic arm mechanism is designed simulated and experimentally tested. A robotic arm is designed in a manner that it can reach the target point provided by user by taking input coordinates from the CT scan images. Designing of this robotic arm is done in SOLIDWORKS 2016 and mathematical equations have been formulated and solved using MATLAB. Optimization of the link length optimization was done using Neural network tool and workspace generation was done using MATLAB. Trajectory generation and path tracing is calculated and simulated using a kinematic model of the real robotic arm in MATLAB. Theoretical accuracy in reaching the target point was found to be fairly good. Experimental model of the robotic arm was setup using stepper motors as joint actuators and ARDUINO MEGA 2560 as microcontroller. Testing was done on the robotic arm by placing an apple in the workspace of the robotic arm and marking an arbitrary target point on that apple. Than the robotic arm was programmed to touch the target point. Level of accuracy found after experimental testing was quite satisfactory.

KEYWORDS: Medical Robotics, Link Length Optimization, Path Tracing, Experimental Model Testing, Solidworks, Matlab \& Arduino
\end{abstract}

Received: Oct 06, 2017; Accepted: Oct 27, 2017; Published: Nov 11, 2017; Paper Id.: IJMPERDDEC201726

\section{INTRODUCTION}

In the assessment and treatment of fatal medical conditions diagnosis plays a vital role in taking first step towards proper medical care. Accurate and timely diagnosis can make a huge difference for a patient and their family. Hence, research is needed to develop the diagnosis field of the medical domain.

In case of suspected tumor or cancer biopsy is done with the help of CT scan images to retrieve a tissue sample from the suspected area for further testing. Currently the limitations in this procedure is that it is performed using eyeball methods and takes longer periods of time. Hence, the accuracy is compromised. In this research an attempt is made to solve this problem by designing a robotic arm that will be able to take target coordinates from the CT scan images as input. Image-guided robotic interventions needs to be accurate, sensitive and easy to use, which primarily focuses on performing minimally invasive operation. This will help doctors perform an accurate biopsy without human errors and it will also reduce the time taken to perform the biopsy. This practically feasible integrated technology approach is highly unique and advantageous for patients and physicians as well. Complications in biopsies will be reduced due to this robotic arm.

This type of robotic technology can also be used in manufacturing industries for engraving, cutting and 
other machining processes with difficult contours.

\section{OBJECTIVE}

The prime objective is to design a robotic arm that can accurately touch the user defined target point placed in its workspace. The robotic arm should also be cost effective and space efficient.

Theoretical analysis and optimization of the link lengths is to be done and kinematic simulation of the robotic arm is done, using Matlab.

Another objective is to manufacture an experimental model of the robotic arm. Testing of the robotic arm is to be done, by marking a target point on an apple, in the workspace of the robotic arm and programming it to reach that, target point. This will help in judging the level of accuracy of the experimental setup, which will then be compared with the theoretical simulation values.

\section{LITERATURE REVIEW}

Francisco Esteban et al, have done a critical analysis on using robotic surgery, for laryngeal surgery concluding that, it was a success. Jihad H. Kaouk et al, have done clinical investigation on a robotic system, for single port urological surgery, by laparoscopic procedure and concluded that, it was successful and post operative pain was much less.

These were a few successful robotic surgeries, but there are many other different types of robotic surgeries that are successful. In a paper published by $\mathrm{C}$ an Acosta Calderon et al, in their research paper "Trajectory generation and tracking of a 5 degree of freedom robotic arm "presented a comparison between an analytical inverse kinematics based hybrid approach and a Resolve Motion Rate Control Method. In a research paper named "Critical Analysis of Robotic Surgery for Laryngeal tumors", by [8] Francisco Esteban et al, they have mentioned the advantages and disadvantages of using the da Vinci robot, for laryngeal tumor surgery and there was only one disadvantage that is being the cost. A research named "A Novel Robotic System for Single-port A number of research reported in the journals C Selcuk Erkaya and Ibrahim Uzmay by using Genetic Algorithm analyzed the effect of the path and the transmission angle due to the presence of joint clearances in a four bar linkage. By using the same Genetic Algorithm Technique, the mechanism was optimized with least error. Research is yet to be done, on the nonlinear behavior of the mechanism. Random variables were assumed such as tolerances and clearances. Mihai Dupac and David G. Beale, examined the mechanism which consists of cracks and clearances. The mechanism was examined with three conditions i.e. (i) no impact (ii) impact on single point and (iii) impact on two points. S. G. Dhande and J. Chakroborthy analyzed a stochastic model of a four bar linkage having path generating linkage. M. J. Tsai and T. H. Lai analyzed the transmission performance of the linkage having joint clearances. Yue-Qing Yu and Bin Jiang compared the mechanism, both analytically and experimentally. The mechanism gave better performance due to the presence of the inertia force. They concluded that, the experimental value satisfy the analytical value.

\section{METHODOLOGY}

\section{Designing of the Robotic Arm}

First step of this research is, to design the robotic arm using a computer aided designing software, such as SOLIDWORKS. The robotic model is designed in Solidworks, using the actual dimensions of the stepper motors that are going to be used in the experimental model. Simulation of motion of each joint was also done in Solidworks, to understand 
the dynamics of the finished robotic arm model.

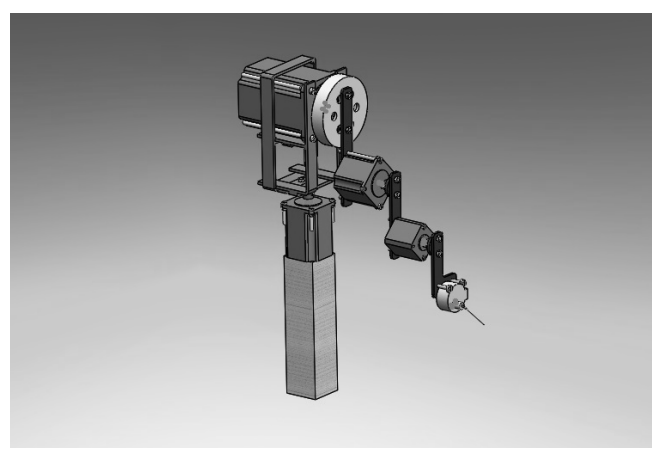

Figure 1: 3D View of the Robotic ar Designed in Solidworks

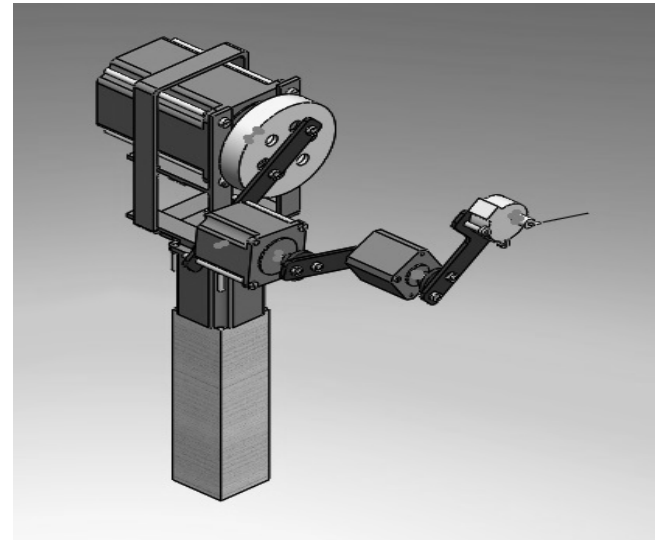

Figure 2: Robotic Arm while Simulating in Solidworks

\section{Nomenclature}

$10=$ length of base, 11 = length of waist link, 12 = length of shoulder link, $13=$ length of arm link, $14=$ length of forearm link, 15 = length of end needle link, $\theta 1=$ orientation angle of the waist link, $\theta 2$ = orientation angle of the shoulder link , $\theta 3=$ orientation angle of the arm link, $\theta 4=$ orientation angle of the forearm link , $\theta 5=$ orientation angle of the end needle link , d2 = Offset between waist link and shoulder link ,d3 = Offset between shoulder link and arm link, d4 = Offset between arm link and forearm link, d5 = Offset between forearm link and end needle, $\mathrm{X}, \mathrm{Y}, \mathrm{Z}=$ Target point coordinates found from CT scan image.

\section{Equation of the Robotic Arm}

Second step was to determine the D-H parameters of the designed robotic arm. For optimized link lengths neural network tool in matlab is used, by taking the workspace as input and the lengths of the links an output. The iterations for training the network was run 100000 times. Link lengths for the robotic arm were taken, according to the graph generated after the training. Forward and inverse kinematics of the robotic arm, were calculated and the equations were used to generate a three dimensional view of the workspace, of this robotic arm using Matlab.

D-H parameter is what defines a robot parameters like its link lengths, joint type, joint angle etc. the below table shows the D-H parameters of this robotic arm. 
Table 1: D-H Parameters of the Robotic Arm

\begin{tabular}{|l|c|c|c|c|}
\hline \multicolumn{1}{|c|}{ Links } & $\mathbf{A}_{\mathbf{i}}(\mathbf{C m}$ & $\mathbf{A}_{\mathbf{i}}$ & $\mathbf{D}_{\mathbf{i}}(\mathbf{C m})$ & $\mathbf{V}_{\mathbf{i}}$ \\
\hline Waist link(1) & 0 & $-90^{0}$ & $\mathrm{l}_{1}=12.35$ & $\theta_{1}$ \\
\hline Shoulder link(2) & $\mathrm{L}_{2}=8.45$ & 0 & $\mathrm{~d}_{2}=-5.9$ & $\theta_{2}$ \\
\hline Arm link(3) & $\mathrm{L}_{3}=7.18$ & 0 & $\mathrm{~d}_{3}=-8.6$ & $\theta_{3}$ \\
\hline Forearm link(4) & $14=6.06$ & 0 & $\mathrm{~d}_{4}=-7$ & $\theta_{4}$ \\
\hline End needle link(5) & $15=4.62$ & 0 & $\mathrm{~d}_{5}=-5.17$ & $\theta_{5}$ \\
\hline
\end{tabular}

The link lengths in the above DH parameters were optimized, using Neural network tool in Matlab. Below Figure.3, is the graph that was obtained after the training of the neural network was completed.

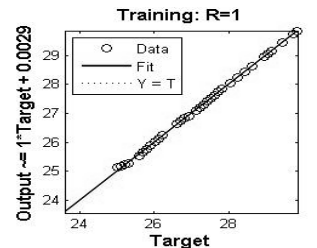

Test: $\mathrm{R}=\mathbf{1}$

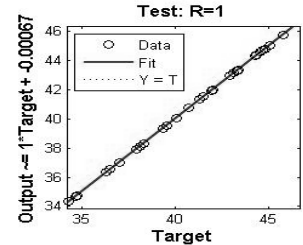

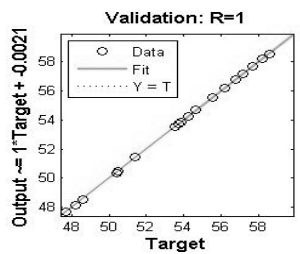

All: $\mathbf{R}=\mathbf{1}$

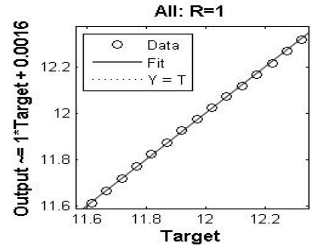

Figure.3: Graph Obtained in Neural Network Tool after the Training of the Network Was Completed

These are the equations, that are formulated using inverse and forward kinematics. $\mathrm{X}, \mathrm{Y}$ and $\mathrm{Z}$ are the target coordinate points, found from CT image.

$\mathrm{X}=\left(\cos \left(\theta_{1}\right) \times \mathrm{l}_{2} \times \cos \left(\theta_{2}\right)\right)-\left(\sin \theta_{1}\left(\mathrm{~d}_{2}+\mathrm{d}_{3}+\mathrm{d}_{4}+\mathrm{d}_{5}\right)\right)+\left(\mathrm{l}_{3} \times \cos \left(\theta_{1}\right) \times \cos \left(\theta_{2}+\theta_{3}\right)\right)+\left(\mathrm{l}_{4} \times \cos \left(\theta_{1}\right) \times \cos \left(\theta_{2}+\theta_{3}+\theta_{4}\right)\right)+\left(1_{5} \times \cos \left(\theta_{1}\right) \times\right.$

$\cos \left(\theta_{2}+\theta_{3}+\theta_{4}+\theta_{5}\right)$

$\mathrm{Y}=\cos \theta_{1}\left(\mathrm{~d}_{2}+\mathrm{d}_{3}+\mathrm{d}_{4}+\mathrm{d}_{5}\right)+\left(\sin \left(\theta_{1}\right) \times \mathrm{l}_{2} \times \cos \left(\theta_{2}\right)\right)+\left(\mathrm{l}_{3} \times \sin \left(\theta_{1}\right) \times \cos \left(\theta_{2}+\theta_{3}\right)\right)+\left(\mathrm{l}_{4} \times \sin \left(\theta_{1}\right) \times \cos \left(\theta_{2}+\theta_{3}+\theta_{4}\right)\right)+\left(\mathrm{l}_{5} \times \sin \left(\theta_{1}\right)\right.$

$\left.\times \cos \left(\theta_{2}+\theta_{3}+\theta_{4}+\theta_{5}\right)\right)$

$\mathrm{Z}=\left(\mathrm{l}_{1}\right)-\left(1_{2} \times \sin \left(\theta_{2}\right)\right)-\left(1_{3} \times \sin \left(\theta_{2}+\theta_{3}\right)\right)-\left(1_{4} \times \sin \left(\theta_{2}+\theta_{3}+\theta_{4}\right)\right)-\left(1_{5} \times \sin \left(\theta_{2}+\theta_{3}+\theta_{4}+\theta_{5}\right)\right)$

This is the transformation matrix of the end needle with respect to the base. 'S' signifies 'sine' and 'C' signifies 'cosine'.

$$
\begin{aligned}
& C \theta_{1} \times C\left(\theta_{2}+\theta_{3}+\theta_{4}+\theta_{5}\right) \quad-C \theta_{1} \times S\left(\theta_{2}+\theta_{3}+\theta_{4}+\theta_{5}\right) \quad-S \theta_{1} \quad X \\
& { }^{0} \mathrm{~T}_{5}=S_{1} \times C\left(\theta_{2}+\theta_{3}+\theta_{4}+\theta_{5}\right) \quad-S \theta_{1} \times S\left(\theta_{2}+\theta_{3}+\theta_{4}+\theta_{5}\right) \quad-C \theta_{1} \quad Y \\
& S\left(\theta_{2}+\theta_{3}+\theta_{4}+\theta_{5}\right) \quad-C\left(\theta_{2}+\theta_{3}+\theta_{4}+\theta_{5}\right) \quad 0 \quad Z \\
& \begin{array}{llll}
0 & 0 & 0 & 1
\end{array}
\end{aligned}
$$

For trajectory planning of the robotic arm a fifth degree polynomial is used, with constraints like (i) Initial position, (ii) Initial velocity (iii) Initial acceleration, (iv) Final position, (v) Final velocity (vi) Final acceleration.

$$
\mathrm{q}(\mathrm{t})=\mathrm{a}_{0}+\mathrm{a}_{1} \mathrm{t}+\mathrm{a}_{2} \mathrm{t}^{2}+\mathrm{a}_{3} \mathrm{t}^{3}+\mathrm{a}_{4} \mathrm{t}^{4}+\mathrm{a}_{5} \mathrm{t}^{5}
$$

t signifies time and $a_{n}(n=0$ to 5$)$ signifies constants.

\section{Workspace of the Robotc Arm}


Figure 4. shows the X-Y-Z 3D view of the work space. In this Figure it is visible that the movement of the robotic arm can be done in 3D space.

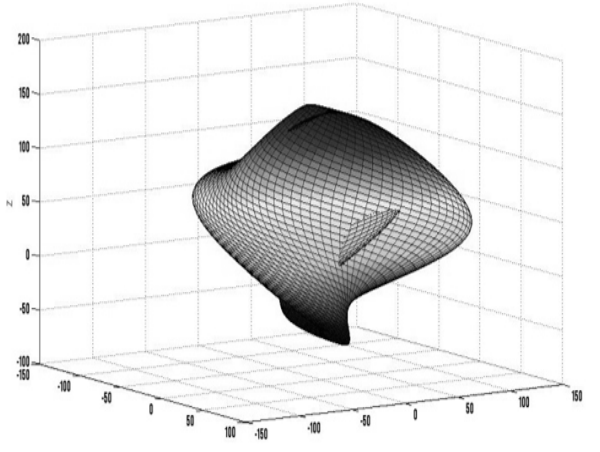

Figure 4: 3D View of Workspace of the Robotic Arm

\section{SIMULATION AND RESULTS}

Third step is to simulate the kinematic model of the robotic arm. by giving an arbitrary target point in its workspace and generating a path for the end needle of the robotic arm to touch at the target point. This was also done using Matlab. There was miniscule deviation that was found, in reaching the target point during simulation, using graphical method. After the simulation angular parameters, for each joint were found.

\section{Path Tracing Trajectory}

The robotic arm traces a curved line when going from one coordinate point to another. There can be more than one ways to reach the target point from the origin point.

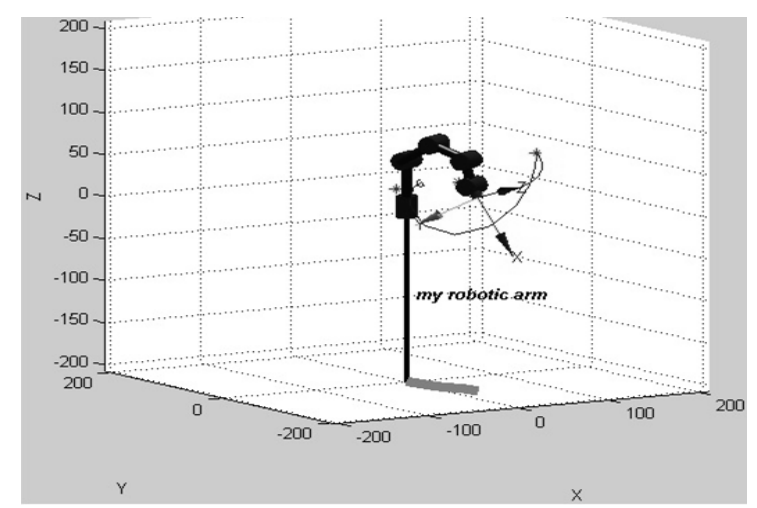

Figure 5: Kinematic Simulation of the Robotic Arm in Matlab

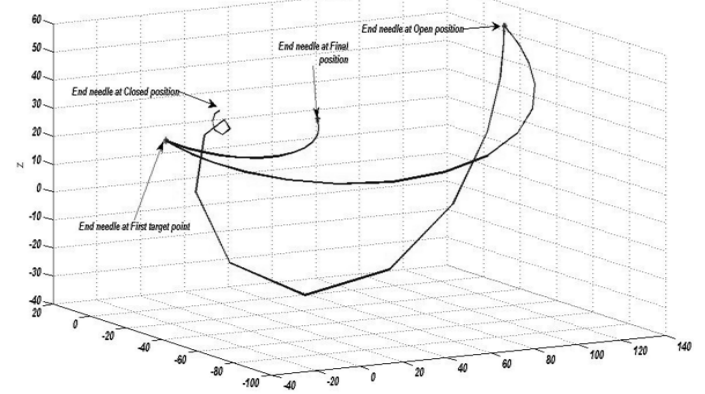




\section{Figure 6: Path Traced by the Robotic Arm after Simulation}

There was a slight deviation in the range of $10^{-4} \mathrm{~mm}$ found after the path was traced in reaching the final target point in curved line trajectory simulation. The theoretical deviation is shown in Table 2.

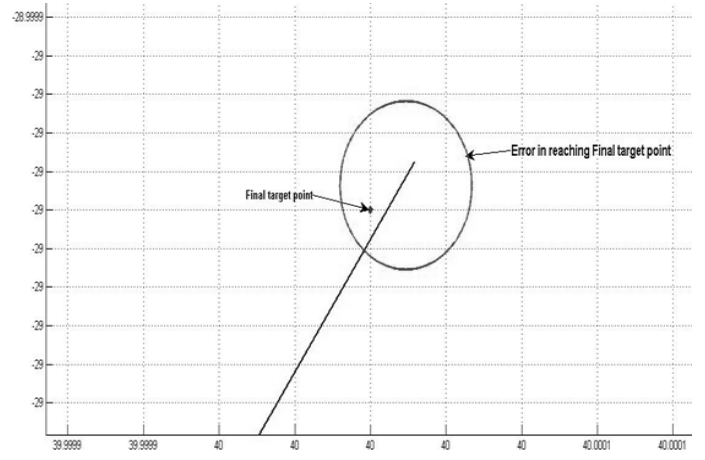

Figure 7: Deviation Found in Tracing a straight Line

\section{EXPERIMENTAL ROBOTIC ARM MODEL}

Experimental model was manufactured using aluminum bars as links for light weight and stepper motors as driving actuators for the robotic arm. The material used for the bade of the robotic arm is cast iron for its heavy weight. Arduino Mega 2560 is used as microcontroller, to control and coordinate the stepper motors at the joints of the robotic arm.

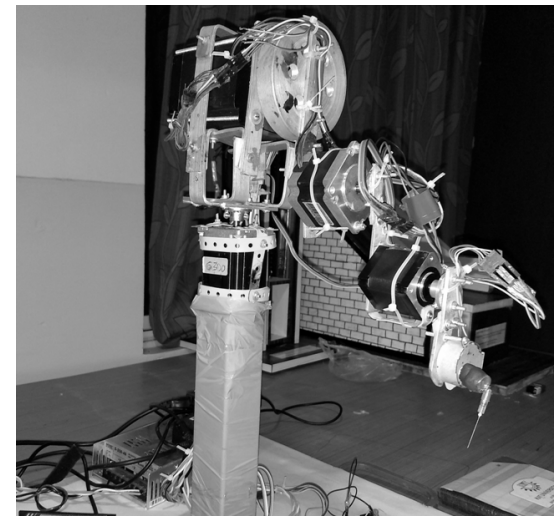

Figure 8: 3D View of the Experimental Setup of the Robotic Arm

\section{Testing of the Experimental Setup}

For testing the accuracy of the robotic arm, an arbitrary point was marked on an apple, which is then placed in the workspace. The robotic arm is than programmed, to reach for that target point on the apple.

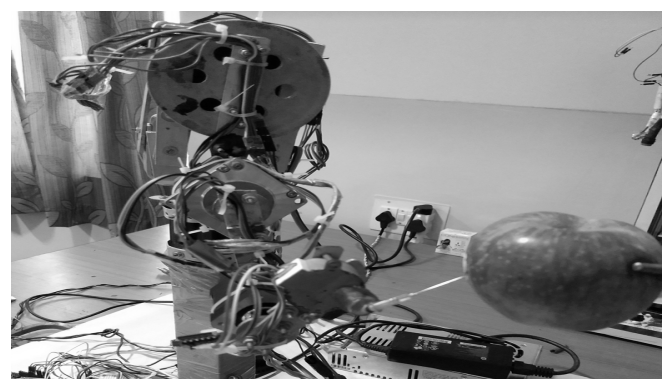

Figure 9: Robotic arm model while it is operational 


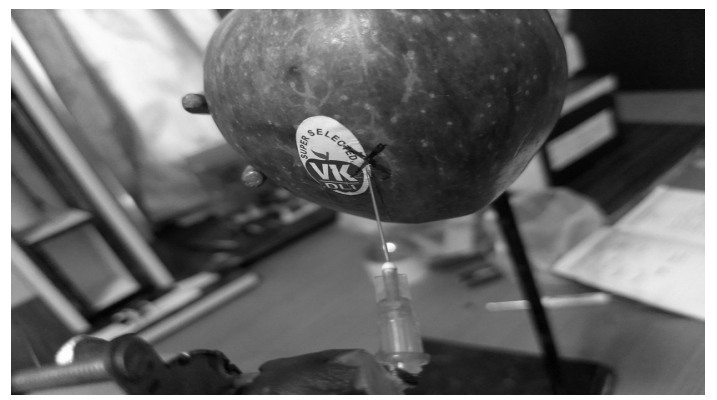

Figure 10: Deviation of the Robotic Arm Model in Reaching the Target Point

There was slight deviation in reaching the target point in experimental analysis but the deviation was found to be well within usable medical parameters. The deviation was primarily due to human errors while modeling the robotic arm and also due to the moderate quality of the stepper motors used as joints. These problems can be easily rectified by properly manufacturing the robotic model and also by using high quality actuators. Below Table 2 is a comparison table of deviation data in analytical simulation and experimental run shown

Table 2: Comparison Table of Deviation Data After Theoretical and Experimental Analysis

\begin{tabular}{|l|c|c|}
\hline Axis & Theoretical Simulation Error & Experimental Error \\
\hline $\mathrm{X}$ & $2.63157 \times 10^{-4} \mathrm{~mm}$ & $0.3 \mathrm{~mm}$ \\
\hline $\mathrm{Y}$ & $5.26310 \times 10^{-5} \mathrm{~mm}$ & $0.5 \mathrm{~mm}$ \\
\hline $\mathrm{Z}$ & $0.00 \mathrm{~mm}$ & $0.9 \mathrm{~mm}$ \\
\hline
\end{tabular}

\section{CONCLUSIONS}

This research showed a space efficient five axis robotic arm that was designed in Solidworks. Link lengths were optimized using neural network tool in matlab and were useful in D-H parameters, equation formulation and experimental modeling of the robotic arm. Kinematic equations of the robotic arm were used, to generate the workspace of the robotic arm. Simulations were done in matlab, by giving an arbitrary point in the workspace and deviation was calculated in each axis, which was in the level of $10^{-4} \mathrm{~mm}$ and is considered to be quite safe.

To validate above results an experimental model was manufactured and Arduino was used, to program the robotic arm model. Testing of the robotic arm was done by placing an apple in the workspace of the robotic arm and marking an arbitrary target point, for the end needle of the robotic arm on that apple.

The experimental testing of the robotic model was successful, as the level of deviation was measured in each axis. The deviation in $\mathrm{X}$ axis was $0.3 \mathrm{~mm}$, in $\mathrm{Y}$ axis was $0.5 \mathrm{~mm}$ and in $\mathrm{Z}$ axis was $0.9 \mathrm{~mm}$. These levels of deviations are quite satisfactory, for the diagnostic domain in medical environment.

The reasons for the deviation in the robotic arm were mostly, due to human error in manufacturing the model and also due to moderate quality of the motors. These problems can be easily rectified by properly manufacturing the robotic model and also by using high quality actuators.

Since it is aimed for medical purpose further research is going to perfect accuracy level of the robotic arm model.

\section{ACKNOWLEDGMENT}

Heartfelt thanks to the affiliated University KIIT, Bhubaneswar, Odisha, India for encouraging and supporting our research and providing adequate resources for the same. 


\section{REFERENCES}

1. Francisco Esteban et al "critical analysis on using robotic surgery for laryngeal surgery".

2. Jihad H. Kaouk et al "clinical investigation on a robotic system for single port urological surgery by laparoscopic procedure”.

3. C an Acosta calderon et al "Trajectory generation and tracking of a 5 degree of freedom robotic arm".

4. S. G. Dhande and J. Chakroborthy, "Analysis and synthesis of mechanical error in linkages-a stochastic approach", Journal of Engineering for Industry, Transactions of the ASME 95 (3) (1973) 672-676

5. M. J. Tsai and T. H. Lai, "Kinematic sensitivity analysis of linkage with joint clearance based on transmission quality", Mechanism and Machine Theory 39 (2004) 1189-1206.

6. Yue- Qing Yu and Bin Jiang, "Analytical and experimental study on the dynamic balancing of flexible mechanisms", Mechanism and Machine Theory 42 (2007), 626-635.

7. Vivek Deshpande \& P M George, Kinematic Modelling and Analysis of 5 DOF Robotic Arm, International Journal of Robotics Research and Development (IJRRD), Volume 4, Issue 2, March - April 2014, pp. 17-24

8. T. G. Boronkay and C. Mei, "Analysis and Design of Multiple Input Flexible Link" Mechanism, Journal of Mechanism 5 (1970) 29-40.

9. Hong-Sen Yan and Ren-chung Soong, "Kinematic and dynamic design of four-bar linkages by links counterweighing with variable input speed", Mechanism and Machine Theory 36 (2001), 1051-1071.

10. A. L. Schwab, J. P. Meijaard and P. Meijer's, "A comparison of revolute joint clearance models in the dynamic analysis of rigid and elastic mechanical system", Mechanism and Machine Theory 37 (2002), 895-913.

11. B. Feng, N. Morita and T. Torii, "A new optimization method for dynamic design of planar linkage with clearances at jointsOptimizing the mass distribution of links to reduce the change of joint forces", Transactions of the ASME (2002).

12. Ruby Mishra, T. K. Naskar and Sanjib Acharya, "Synthesis of coupler curve of a four bar linkage with joint clearances", International Journal of Engineering Research and Application(IJERA), ISSN:2248-9622 (2013), 1193-1199.

13. Ruby Mishra, T. K. Naskar and Sanjib Acharya, "Path synthesis of four-bar linkages with joint clearances using DE algorithm", Proceedings of the National Conference on Trends and Advances in Mechanical Engineering(2012).

14. K. W. Ting, J. Zhu and D. Watkins, "The effect of joint clearance on position and orientation deviation of linkages and manipulators", Mechanism and Machine Theory 35 (2000), 391-401 